\title{
The Ability to Detoxify the Mycotoxin Deoxynivalenol Colocalizes With a Major Quantitative Trait Locus for Fusarium Head Blight Resistance in Wheat
}

\author{
Marc Lemmens, ${ }^{1}$ Uwe Scholz, ${ }^{1}$ Franz Berthiller, ${ }^{2}$ Chiara Dall'Asta, ${ }^{2}$ Andrea Koutnik, ${ }^{1}$ \\ Rainer Schuhmacher, ${ }^{2}$ Gerhard Adam, ${ }^{3}$ Hermann Buerstmayr, ${ }^{1}$ Ákos Mesterházy, ${ }^{4}$ Rudolf Krska, ${ }^{2}$ \\ and Peter Ruckenbauer ${ }^{1}$ \\ ${ }^{1}$ Institute for Plant Production Biotechnology, and ${ }^{2}$ Center for Analytical Chemistry, Department IFA-Tulln, BOKU-University \\ of Natural Resources and Applied Life Sciences, Konrad Lorenzstr. 20, A-3430 Tulln, Austria; ${ }^{3}$ Institute of Applied Genetics \\ and Cell Biology, Department of Applied Plant Sciences and Plant Biotechnology, BOKU-University \\ of Natural Resources and Applied Life Sciences, Muthgasse 18, A-1190, Vienna, Austria; ${ }^{4}$ Laboratory of Plant Pathology, \\ Wheat Breeding Department, Cereal Research non-profit Co., Alsókikötösor 9, 6701, Szeged, Hungary
}

Submitted 5 June 2005. Accepted 15 August 2005.

\begin{abstract}
We investigated the hypothesis that resistance to deoxynivalenol (DON) is a major resistance factor in the Fusarium head blight (FHB) resistance complex of wheat. Ninety-six double haploid lines from a cross between 'CM-82036' and 'Remus' were examined. The lines were tested for DON resistance after application of the toxin in the ear, and for resistances to initial infection and spread of FHB after artificial inoculation with Fusarium spp. Toxin application to flowering ears induced typical FHB symptoms. Quantitative trait locus (QTL) analyses detected one locus with a major effect on DON resistance (logarithm of odds $=\mathbf{5 3 . 1}$, $R^{2}=92.6$ ). The DON resistance phenotype was closely associated with an important FHB resistance QTL, Qfhs.ndsu$3 B S$, which previously was identified as governing resistance to spread of symptoms in the ear. Resistance to the toxin was correlated with resistance to spread of FHB $(r=$ $0.74, P<0.001)$. In resistant wheat lines, the applied toxin was converted to $\mathrm{DON}-3-O$-glucoside as the detoxification product. There was a close relation between the DON-3glucoside/DON ratio and DON resistance in the toxintreated ears $\left(R^{2}=0.84\right)$. We conclude that resistance to DON is important in the FHB resistance complex and hypothesize that Qfhs.ndsu-3BS either encodes a DON-glucosyltransferase or regulates the expression of such an enzyme.
\end{abstract}

Fusarium head blight (FHB) of wheat can severely reduce grain yield and quality. A complex of fungal species of the genus Fusarium causes the disease. The most important causal fungi in Europe and North America are Fusarium graminearum Schwabe and F. culmorum (W. G. Sm.) Sacc. These produce trichothecene mycotoxins, predominantly deoxynivalenol (DON) (Bottalico and Perrone 2002; McMullen et al. 1997), that are potent inhibitors of protein synthesis in eukaryotes (McLaughlin et al. 1977) and are also phytotoxic, causing chlorosis, necrosis, and wilting in planta. The production of trichothecenes is considered a virulence factor of several Fusa-

Corresponding author: M. Lemmens; Telephone: (+43)-(0)2272-66280205; Fax: (+43)-(0)2272-66280-203; E-mail: marc.lemmens@ boku.ac.at

Current address of C. Dall'Asta: Dipartimento di Chimica Organica e Industriale, Parco Area delle Scienze 17/A, I-43100 Parma, Italy. rium spp. Disruption of the trichothecene biosynthetic pathway in $F$. graminearum and subsequent artificial inoculation experiments in the greenhouse and the field showed that the trichothecene-nonproducing $F$. graminearum mutants were still pathogenic but less virulent, being unable to spread from the inoculated spikelet through the ear like the wild type (Bai et al. 2001; McCormick 2003). Trichothecenes may suppress the expression of plant defense proteins induced after host infection. Therefore, we suspected that molecular mechanisms conferring trichothecene resistance might be important components of the polygenic FHB resistance trait. It previously was postulated that wheat lines resistant to these toxins would not show complete resistance but would show an increased resistance to spread of symptoms in the host and inherent prevention of trichothecene accumulation (Miller and Greenhalgh 1988; Snijders 1994). However, conclusive evidence that DON resistance exists in wheat and significantly contributes to FHB resistance in planta is still missing.

Total FHB resistance in wheat can be dissected into several resistance components (Mesterházy et al. 1999). Resistances to initial infection (resistance component type I) and spread of FHB in the host (type II) have been identified (Schroeder and Christensen 1963) and are commonly accepted. Resistance component type III was described as insensitivity of wheat lines to the toxin, defined as the ability of the resistant cultivar to degrade DON (Wang and Miller 1988). It is not clear whether and to what extent FHB-resistant lines possess resistance to DON. Bioassays and plant tissues used for toxin resistance tests to date have been diverse: growth inhibition of shoots and roots (Shimada and Otani 1990) or calli (Bruins et al. 1994), inhibition of seed germination (Lemmens et al. 1994; Wakulinski 1989), coleoptile growth retardation (Wang and Miller 1988), and electrolyte leakage induced by DON in cereal leaves (Wojciechowski et al. 1995). Attempts to correlate DON resistance to Fusarium resistance have yielded variable results. Some authors describe a significant correlation between toxin resistance and field FHB resistance (Lemmens et al. 1994; Wang and Miller 1988); others did not find a close relation between the two variables (Bruins et al. 1994). The reasons for these contradictory results are not clear; however, factors such as the stage of plant development, the tissue studied, and concentration and delivery of the toxin may be important in determining when and where DON is relevant in the disease 
process (McCormick 2003). FHB-resistant wheat lines derived from different genetic resources may possess different FHB resistance genes (Mesterházy 2003), and DON resistance might be detectable only in certain populations segregating for FHB resistance.

Biochemical mechanisms of resistance to DON, such as catabolism and conjugation of DON, have been reported. Callus cultures of the FHB-resistant wheat cv. Frontana converted more ${ }^{14} \mathrm{C}$-labeled DON into uncharacterized products than callus derived from the susceptible wheat cv. Casavant (Miller and Arnison 1986). The authors provided evidence that the trichothecene was converted to a glucoside (Miller and Arnison 1986; Miller and Greenhalgh 1988), but this metabolite was not characterized further. Recently, it has been shown that, in Arabidopsis thaliana, the conjugation product of DON is DON-3-O-glucoside, which, when compared with DON, had a strongly reduced ability to inhibit protein synthesis by a wheat germ extract in vitro (Poppenberger et al. 2003).

The goal of this work was to gain insight into the role of DON resistance in the FHB resistance complex. To this end, we developed a new method to evaluate DON resistance in wheat. We found significant correlations between DON resistance and Fusarium resistance data in a segregating double haploid (DH) wheat population. Further, in investigating the biochemical mechanism of resistance to DON, we were able to demonstrate that the ability to efficiently detoxify DON into a DON-glucose conjugate co-localizes with a previously identified major FHB resistance quantitative trait locus (QTL).

\section{RESULTS}

Water-soaked spots resembling early FHB symptoms were the first noticeable symptoms caused by DON on wheat. These spots appeared in less than $1 \%$ of the DON-treated spikelets 2 to 4 days after treatment. The toxin-treated spikelets of DONsensitive wheat lines developed a typical straw-like color 5 to 7 days post treatment. These bleaching symptoms spread in

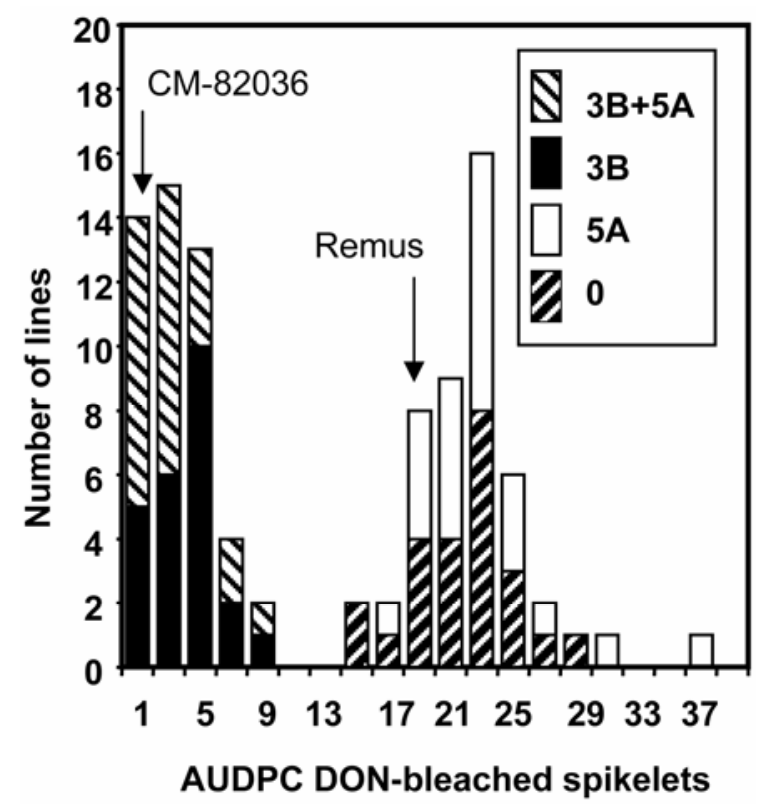

Fig. 1. Frequency distribution of 96 wheat lines for mean area under the disease progress curve values due to DON damage $\left(\mathrm{AUDPC}_{\mathrm{DON}}\right)$. Values are means over two seasons and two replications. Values of parental lines are indicated by arrows. The least significant difference for comparison of line means $(\alpha=0.05)$ was 7.4. 3B+5A, lines with both Fusarium head blight quantitative trait loci; 3B, lines with $Q f h s . n d s u-3 B S$ only; $5 \mathrm{~A}$, lines with $Q$ fhs.ifa-5A only; 0 , lines lacking both quantitative trait loci. both acropetal and basipetal directions. Wilting of the complete ear above point of treatment was observed in less than $1 \%$ of the treated ears. Spread of symptoms ceased 16 days after DON treatment. In sensitive lines, there was no kernel set in the ear above point of treatment, even when no bleaching or wilting symptoms were detected in this part of the ear. As expected, kernels showed no FHB symptoms such as the pinkorange pigmentation caused by fungal mycelium. Ears treated with the control solution developed normally.

Analysis of variance (ANOVA) analyses with the area under the disease progress curve (AUDPC) data of fungal disease parameters and of DON resistance parameters showed that highly significant differences existed between wheat lines. There was no significant year-by-genotype interaction for DON resistance and the data of both seasons were highly correlated $(r=0.90, P<0.001)$. The frequency distribution of the mean AUDPC values over two seasons for DON resistance $\left(A U D P C_{D O N}\right)$ showed two clearly distinguishable peaks (Fig. 1). The least significant difference for comparison of line means $(\alpha=0.05)$ was 7.4. The AUDPC levels of 'CM-82036' and 'Remus' were 0.7 and 21.2 AUDPC units, respectively. QTL class means were 2.8 (24 wheat lines with both FHB QTL), 3.9 (24 lines with $Q f h s . n d s u-3 B S$ only), 22.9 (24 lines with $Q f h$ s.ifa-5A only), and 21.7 AUDPC units for the 24 lines lacking both QTL. Least significant difference for comparison of QTL class means was 1.6 AUDPC units $(\alpha=0.05)$. The wheat lines with $Q f h s . n d s u-3 B S$ showed a high resistance level against DON. Qfhs.ifa-5A had no association with DON resistance.

To study the genetic background of DON resistance in more detail, independent QTL analyses were done using DON resistance data from the 96 wheat lines. Interval mapping using simple interval mapping (SIM) and composite interval mapping (CIM) detected one prominent effect on chromosome $3 \mathrm{~B}$ associated with Xbarc147. Results of the CIM analyses are summarized in Table 1. Logarithm of odds values and explained phenotypic variance tended to reach a maximum using DON resistance data assessed 13 and 16 days post treatment. DON resistance mapped to the same region on chromosome $3 \mathrm{~B}$ as FHB resistance (Fig. 2). No other QTL for toxin resistance were detected in this wheat population.

DON resistance data were compared with FHB resistance data and the results of correlation analyses are summarized in Table 2. FHB resistance data were generated with two strains of DON-producing Fusarium spp. Total FHB resistance was evaluated as the percentage of bleached spikelets (AUDPC severity $_{\text {). }}$. In addition, resistances to initial infection and to spread of FHB symptoms were assessed individually. Disease incidence (AUDPC $\mathrm{incidence}_{\text {e }}$ ) was taken as a measure of resistance to initial infection. Two separate parameters were evaluated as a measure

Table 1. Quantitative trait loci for deoxynivalenol (DON) resistance ${ }^{\mathrm{a}}$

\begin{tabular}{lcccc}
\hline $\begin{array}{l}\text { DON } \\
\text { resistance }\end{array}$ & Marker interval & Chromosome & LOD & VE \\
\hline NDBS $_{7}$ & Xbarc147-Xgwm493 & 3B & 17.8 & 58.3 \\
NDBS $_{10}$ & Xbarc147-Xgwm493 & 3B & 35.7 & 82.6 \\
NDBS $_{13}$ & Xbarc147-Xgwm493 & 3B & 52.6 & 92.4 \\
NDBS $_{16}$ & Xbarc147-Xgwm493 & 3B & 53.1 & 92.6 \\
AUDPC $_{\text {DON }}$ & Xbarc147-Xgwm493 & 3B & 47.1 & 90.1 \\
\hline
\end{tabular}

a Analyses were done with composite interval mapping. The number of DON-bleached spikelets $\left(\mathrm{NDBS}_{x}\right)$ in a basipetal direction, including the treated spikelets, was assessed at 7, 10, 13, and 16 days after the first treatment. Data were used to calculate the area under the disease progress curve $\left(\mathrm{AUDPC}_{\mathrm{DON}}\right)$. Further results on chromosome location, logarithm of odds (LOD), and the percentage of explained phenotypic variance (VE) are presented for the mean data across the 2 years of investigation. 
of resistance to spread of FHB symptoms: number of bleached spikelets (AUDPC bleaching $_{\text {) }}$ and percentage of wilted ears $\left(\mathrm{AUDPC}_{\text {wilting }}\right)$. DON resistance data showed only a weak correlation with $\mathrm{AUDPC}_{\text {severity }}$ and no significant correlation with $\mathrm{AUDPC}_{\text {incidence }}$. Both $\mathrm{AUDPC}_{\text {bleaching }}$ and $\mathrm{AUDPC}_{\text {wilting data }}$ were highly correlated with DON resistance parameters $(r=$ 0.51 to $0.74, P<0.001$ in all cases). Correlation coefficients tended to reach a maximum using DON resistance data assessed 13 and 16 days post treatment. We conclude that DON resistance was associated with resistance to spread of FHB, but not with resistance to initial infection.

Toxin analyses were done for 32 randomly selected DH lines: 9 lines with both QTL for FHB resistance, 6 lines with Qfhs.ndsu-3BS only, 11 lines with $Q f h s . i f a-5 A$ only, and 6 lines lacking both QTL. Neither trichothecenes nor DON conjugates were detected in ears treated with control solution. In toxintreated ears of all investigated lines, DON and DON-3- $O$ -
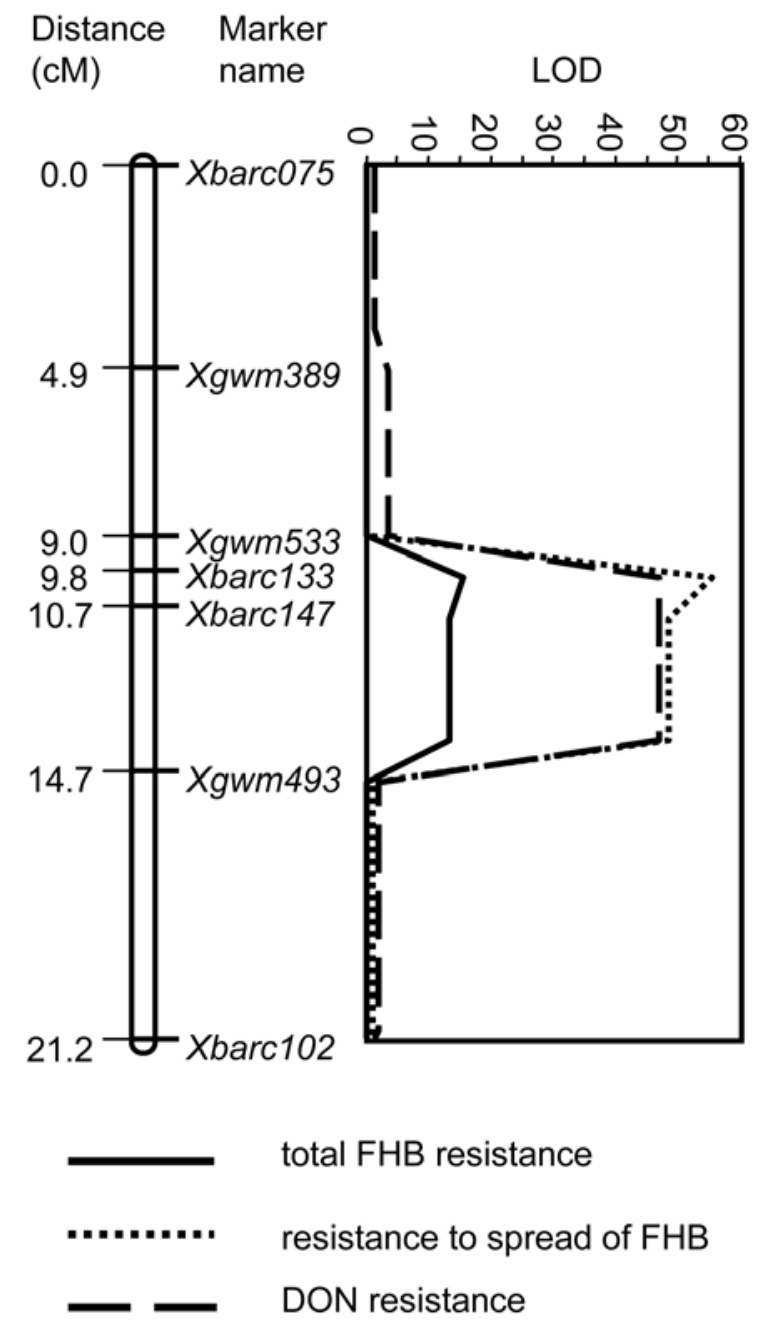

Fig. 2. Interval analysis of a quantitative trait locus for total Fusarium head blight (FHB) resistance, resistance to spread of FHB (type II resistance), and deoxynivalenol (DON) resistance on a linkage group corresponding to part of chromosome 3BS. At the left: genetic map of part of chromosome 3BS (distance between markers in centimorgans [cM]). At the right: logarithm of odds (LOD) curves for total FHB resistance (full line), resistance to spread of FHB symptoms (dotted line), and DON resistance (dashed line). The parameters used for calculations were area under the

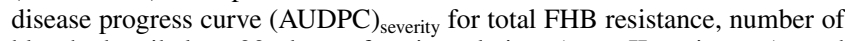
bleached spikelets 22 days after inoculation (type II resistance), and $\mathrm{AUDPC}_{\mathrm{DON}}$ (DON resistance). Data are means over two growth seasons in all cases. LOD curves were calculated with composite interval mapping. The linkage map is based on Buerstmayr and associates (2003). glucoside were present. DON concentrations in ears of different DH lines varied from 32 to $455 \mathrm{mg} / \mathrm{kg}$ and DON-3-Oglucoside concentrations ranged from 36 to $507 \mathrm{mg} / \mathrm{kg}$. The relation between DON resistance and ratios of DON-3-O-glucoside to DON concentrations in toxin-treated ears is illustrated in Figure 3. The data were fitted to a logarithmic curve. The coefficient of determination $\left(R^{2}\right)$ was 0.84 . DH lines with Qfhs.ndsu-3BS converted more DON to DON-3-O-glucoside, resulting in a high DON-glucoside/DON ratio and a high DON resistance level.

\section{DISCUSSION}

In this study, we wanted to provide evidence for the existence of resistance to DON in planta and to evaluate the importance of DON resistance in the FHB resistance complex. Furthermore, we aimed to gain insight into the DON detoxification mechanisms.

A first essential step toward reaching these goals was the development of a bioassay that allowed quantification of DON resistance. The assay that we have developed is based on the application of DON to flowering wheat ears. The flowering ear was chosen because this developmental stage and tissue is the main target of Fusarium spp. causing FHB (Sutton 1982). We also have studied DON resistance in the same wheat population using other bioassays, such as seed germination and DON-induced electrolyte leakage from flag leaf disks (data not shown). Although these tests also discriminated highly significant differences in the DON resistance of these wheat lines, the data showed no significant correlation with either the DON resistance data in the ear or with the FHB resistance data assessed after artificial inoculation with the fungus (unpublished data).

In this study, DON was applied directly to the floret between palea and lemma. The toxin had to diffuse into the plant tissue. A detergent was added in the test solutions to improve wetting and diffusion. The relative air humidity in the growth cabinet was raised to $80 \%$ during toxin application to slow down evaporation of the applied droplet, thus prolonging the time available for diffusion. In sensitive wheat lines, there was no kernel set from the point of DON application upward, even when no bleaching or wilting symptoms were observed in this part of the ear. Therefore, we assume that DON is quickly taken up and transported in the apoplast, including the xylem. Additional tests (results not shown) revealed that less than $5 \%$ of the total trichothecene amount measured in the ear (DON + DON-glucoside) could be washed off from the treated spikelets, indicating that a large proportion of the DON applied was taken up in the ear. In resistant lines, the majority of the DON was converted to DON-3-O-glucoside; therefore, we can exclude the possibility that DON resistance observed was due to inhibition or lack of uptake of the toxin in planta. A total of $1 \mathrm{mg}$ of DON was applied to each toxin-treated ear. The highest wholeear DON level measured was $455 \mathrm{mg} / \mathrm{kg}$. After natural infection, DON at up to $600 \mathrm{mg} / \mathrm{kg}$ has been reported in single tombstone kernels (Sinha and Savard 1997), indicating that such high local DON concentrations occur in planta.

A second essential step for the success of this work was the choice of the mapping population under investigation and the extensive field investigations on specific FHB resistance components toward the fungus. CM-82036 was the resistant parent in the mapping population. This mapping population has been intensively investigated and two major QTL for FHB resistance have been mapped. CM-82036, originating from a cross 'Sumai-3'/'Thornbird-S', showed a high level of resistance to initial infection, spread of FHB symptoms (Buerstmayr 2002, 2003), and DON. Other experiments (results not shown) con- 
firmed the high DON resistance level of Sumai-3. The DH population was investigated thoroughly for total FHB resistance as well as for resistance to initial infection and to spread of FHB. These were assessed individually in the field using specific artificial inoculation techniques. Although resistance to spread of symptoms is important in total FHB resistance, DON resistance data did not relate well to total FHB resistance. This highlights the importance of the assessment of individual resistance components.

It was postulated that wheat lines resistant to DON would show increased resistance to colonization (Miller and Greenhalgh 1988; Snijders 1994). Infections with Tri5--mutants demonstrated that the trichothecene-nonproducing $F$. graminearum mutants were unable to spread from the inoculated spikelet through the ear like the wild type (Bai et al. 2001). They concluded that DON is a virulence factor with an important role in the spread of FHB in the ear. If in planta DON resistance exists, we expected it to play a main role in resistance to spread of FHB. According to our data, DON resistance was, indeed, closely correlated with resistance to spread. This indicates that DON resistance is i) an important part of the FHB resistance complex and ii) probably an integral part of the resistance to spread of $\mathrm{FHB}$, acting by inhibiting growth of the fungus in the ear. If we consider the highest correlation coefficient between DON resistance data and data for resistance to spread of FHB ( $r=0.74$ ) (Table 2$)$, we can explain approximately $50 \%$ of the observed variation at best. Therefore, we postulate that additional fungal virulence mechanisms exist, and other as-yet-unknown resistance mechanisms contribute to resistance to spread of FHB symptoms in the wheat ear.

DON resistance and the major QTL for FHB resistance, Qfhs.ndsu-3BS, mapped to the same region on chromosome 3B. Qfhs.ndsu-3BS is an important QTL for FHB resistance (Waldron et al. 1999) and explains $29 \%$ of the phenotypic variance for visual FHB severity in the CM-82036/Remus population (Buerstmayr et al. 2003) and $41.6 \%$ of the resistance to spread of FHB in a Sumai-3/'Stoa' population (Anderson et al. 2001). This provides additional evidence for the important role of DON resistance in the total FHB resistance complex. In our wheat population, resistance to DON was monogenic and the gene had a major effect. In wheat, other QTL associated with low DON concentration following artificial inoculation have been reported. QTL controlling the accumulation of DON were detected on chromosome 2DS and 5AS in a DH population originating from a cross between 'Wuhan-1' and 'Maringa' (Somers et al. 2003). These QTL differ from the one described in this article, and did not improve FHB resistance.

A third essential step to reach our goals was the identification of DON-3-O-glucoside as a detoxification product of DON in wheat. In DON-resistant lines, the majority of the applied
DON was conjugated to glucose. The close relation between the ratio of DON-3-O-glucoside to DON concentration in ears with the DON resistance data $\left(R^{2}=0.84\right)$ strongly suggests that DON conjugation to glucose is the primary biochemical mechanism for resistance towards DON. This observation and the fact that $Q f h s . n d s u-3 B S$ governs DON resistance led to the hypothesis that $Q f h s . n d s u-3 B S$ either encodes a glucosyl transferase or regulates the expression of such an enzyme.

For practical breeding, these findings might have several consequences. We know that the DON-producing ability of the Fusarium isolates correlates well with their virulence (Mesterházy 1995, 2002). It is expected that the introduction of DON resistance to wheat will reduce pathogen virulence and will increase total FHB resistance, leading to fewer symptoms and lower DON contamination levels relative to DONsusceptible wheat cultivars. Indeed, the DON conjugate formed in DON-resistant lines was found in both artificially inoculated and naturally infected wheat samples (Berthiller et al. 2005). Results obtained with a small set of wheat lines revealed that, although resistant cultivars contained higher amounts of DON-glucoside, total content of DON + DON-glu-

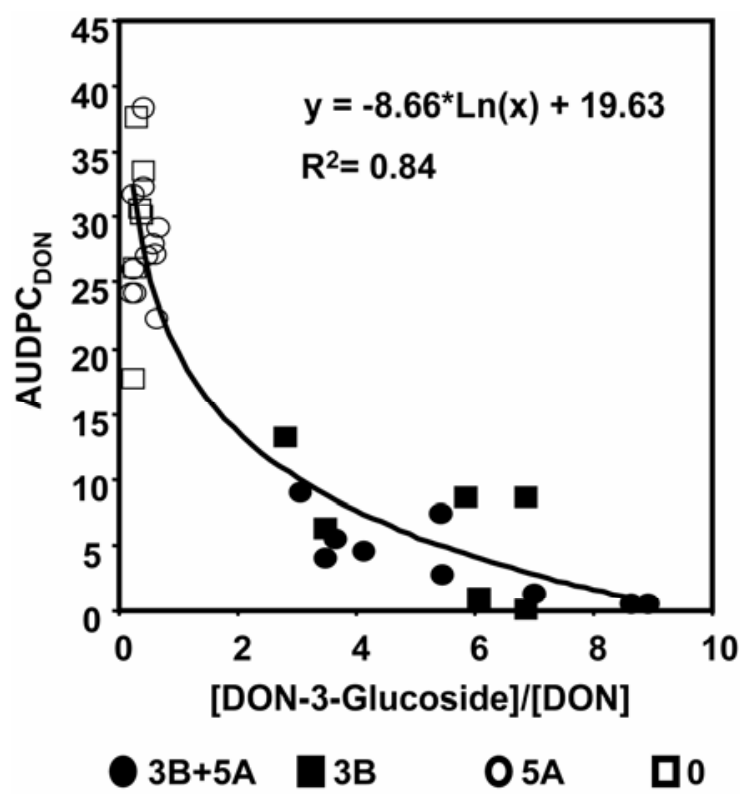

Fig. 3. Relation between deoxynivalenol (DON) resistance (area under the disease progress curve $[\mathrm{AUDPC}]_{\mathrm{DON}}$ ) and the ratio of the concentrations of DON-3-glucoside to DON in the toxin treated ears. Filled circles, double haploid lines with $Q f h s . i f a-5 A$ and $Q f h s . n d s u-3 B S$; filled squares, lines with $Q f h s . n d s u-3 B S$ only; open circles, wheat lines with $Q f h s . i f a-5 A$ only; open squares, lines lacking both quantitative trait loci.

Table 2. Correlation coefficients between the data for Fusarium head blight (FHB) and deoxynivalenol (DON) resistance ${ }^{\text {a }}$

\begin{tabular}{|c|c|c|c|c|c|c|c|c|}
\hline \multirow[b]{2}{*}{ Resistance $^{b}$} & \multicolumn{2}{|c|}{ AUDPC $_{\text {severity }}$} & \multicolumn{2}{|c|}{ AUDPC $_{\text {incidence }}$} & \multicolumn{2}{|c|}{ AUDPC $_{\text {bleaching }}$} & \multicolumn{2}{|c|}{ AUDPC $_{\text {wilting }}$} \\
\hline & F. culmorum & $F$. graminearum & F. culmorum & F. graminearum & F. culmorum & F. graminearum & F. culmorum & F. graminearum \\
\hline $\mathrm{NDBS}_{7}$ & $0.06 \mathrm{~ns}$ & $0.18 \mathrm{~ns}$ & $-0.12 \mathrm{~ns}$ & $-0.03 \mathrm{~ns}$ & $0.54 * * *$ & $0.51 * * *$ & $0.61 * * *$ & $0.58 * * *$ \\
\hline $\mathrm{NDBS}_{10}$ & $0.16 \mathrm{~ns}$ & $0.30 * *$ & $-0.05 \mathrm{~ns}$ & $0.08 \mathrm{~ns}$ & $0.61 * * *$ & $0.62 * * *$ & $0.62 * * *$ & $0.65 * * *$ \\
\hline $\operatorname{NDBS}_{13}$ & $0.24 *$ & $0.37 * * *$ & $-0.01 \mathrm{~ns}$ & $0.13 \mathrm{~ns}$ & $0.68 * * *$ & $0.70^{* * *}$ & $0.67 * * *$ & $0.73 * * *$ \\
\hline $\mathrm{NDBS}_{16}$ & $0.24 *$ & $0.36 * * *$ & $-0.01 \mathrm{~ns}$ & $0.12 \mathrm{~ns}$ & $0.69 * * *$ & $0.71 * * *$ & $0.69 * * *$ & $0.74 * * *$ \\
\hline $\mathrm{AUDPC}_{\mathrm{DON}}$ & $0.19 \mathrm{~ns}$ & $0.33 * * *$ & $-0.05 \mathrm{~ns}$ & $0.09 \mathrm{~ns}$ & $0.66 * * *$ & $0.68 * * *$ & $0.68 * * *$ & $0.71 * * *$ \\
\hline
\end{tabular}

${ }^{a}$ FHB resistance parameters were the area under the disease progress curve (AUDPC) data for disease severity, disease incidence, number of bleached spikelets, and percentage of wilted ears $\left(\mathrm{AUDPC}_{\text {severity }}, \mathrm{AUDPC}_{\text {incidence }}, \mathrm{AUDPC}_{\text {bleaching }}\right.$, and $\mathrm{AUDPC}$ wilting, respectively). DON resistance data were number

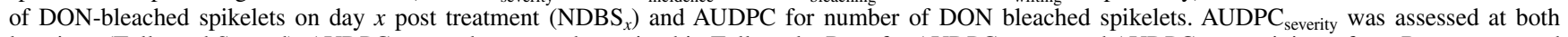

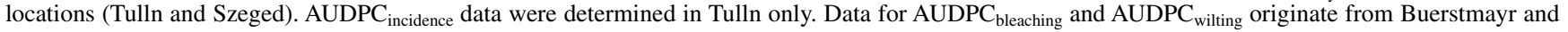
associates (2002). All resistance data are means over 2 years; $* * * * *$, and $*=$ significant at $P<0.001,0.01$, and 0.05 , respectively; ns $=$ not significant $(P>$ $0.05)$.

${ }^{\mathrm{b}}$ DON resistance in 2003 and 2004. 
coside is an order of magnitude larger in DON- and Fusariumsusceptible wheat cultivars (Berthiller et al. 2005). The biochemical fate of DON-3-O-glucoside in human and animal intestinal tracts is currently unclear. DON-3-O-glucoside produced in DON-resistant wheat lines could be a so-called "masked mycotoxin" (Gareis et al. 1990), which is not detectable with routine analyses but which might regain its biological activity after the glucose moiety is cleaved off in the intestinal tract. In this respect, it is interesting to mention that $\mathrm{cv}$. Sumai-3, which contains the major QTL for FHB resistance, Qfhs.ndsu-3BS, is the most widely used resistance source in the world (Mesterházy 2003).

It generally is accepted that resistance to FHB is non-species specific (i.e., QTL for FHB resistance are equally effective against all Fusarium strains irrespective of Fusarium spp.) (Parry et al. 1995). It is not clear, at present, whether the postulated glucosyl transferase can detoxify other trichothecenes (e.g., nivalenol, 15-acetyl-DON, and 3-acetyl-DON) or even other mycotoxins (e.g., moniliformin). The cloned Arabidopsis DOGTl glucosyl transferase, for instance, has a fairly narrow substrate specificity and is active against nivalenol only at low temperatures (G. Adam, unpublished data). Hence, the intriguing possibility exists that $Q f h s . n d s u-3 B S$ encodes a glucosyl transferase that is effective only against Fusarium strains that produce DON or structurally highly similar trichothecenes. In this scenario, resistance to DON in wheat could be a Fusarium-spp.-specific or strain-specific trait that potentially could be overcome by chemotypes producing structural variants of DON. This hypothesis is currently under investigation at our institute using near-isogenic wheat lines.

On the other hand, it seems quite reasonable to speculate that $Q f h s . n d s u-3 B S$ encodes a classical resistance gene that recognizes a Fusarium-derived elicitor (DON in our investigations) and triggers the induction of a battery of genes, including one or several members of the very large gene family of glucosyl transferases (Bowles 2002), which collectively antagonize DON and other fungal toxins.

\section{MATERIALS AND METHODS}

\section{Plant material.}

A population of 96 recombinant $\mathrm{F}_{1}$-derived $\mathrm{DH}$ lines was selected for these investigations. The lines originate from a cross between CM-82036 (highly resistant to FHB) and Remus (susceptible) (Buerstmayr et al. 2002). CM-82036 originated from the cross Sumai-3 and Thornbird. CM-82036 carries two major QTL for FHB resistance ( $Q f h s . n d s u-3 B S$ and $Q f h s . i f a-5 A)$ (Buerstmayr et al. 2003). All four QTL classes resulting from all possible combinations of these QTL were present in the selected nursery (24 lines in each class, including the parents).

\section{Field inoculations and field disease parameters.}

The wheat lines were tested for total FHB resistance (= sum of all FHB resistance components) and for individual FHB resistance components (resistance to initial infection and to spread of FHB symptoms) during two growth seasons at the experimental field at Tulln, Austria. All Fusarium isolates used for the artificial inoculations were DON producers. Field plots were located near the Danube river $30 \mathrm{~km}$ west of Vienna, Austria $\left(48^{\circ} 20^{\prime} \mathrm{N}, 16^{\circ} 4^{\prime} \mathrm{E}, 180 \mathrm{~m}\right.$ above sea level, $9.2^{\circ} \mathrm{C}$ mean annual temperature, $620 \mathrm{~mm}$ of annual rainfall). Agronomy was done as described before (Buerstmayr et al. 2002). Total FHB resistance was determined at a second field near Szeged, Hungary $\left(46^{\circ} 15^{\prime} \mathrm{N}, 20^{\circ} 10^{\prime} \mathrm{E}, 80 \mathrm{~m}\right.$ above sea level, $10.5^{\circ} \mathrm{C}$ mean annual temperature, $580 \mathrm{~mm}$ of annual rainfall).

To determine total FHB resistance and resistance to initial infection, the lines were spray inoculated in 2002 and 2003 in
Tulln. Experimental layout was a split-plot design (main plot was genotype and subplot was Fusarium isolate) with two replications. Lines were sown in $2-\mathrm{m}^{2}$ plots. The $\mathrm{DH}$ population was tested with $F$. graminearum and $F$. culmorum strains (IFA205 and IFA211, respectively). Conidial suspensions in final concentrations of $10^{5} / \mathrm{ml}$ were prepared. Inoculations and visual assessments were carried out exactly as described previously (Buerstmayr et al. 2003). Disease severity, estimated as percentage of diseased spikelets, was taken as a measure of total FHB resistance. Total FHB resistance combines all possible resistance components, including resistance to initial infection and spread of FHB and DON (and probably additional resistance components). Disease incidence was assessed by determining the percentage of ears showing FHB symptoms and was taken as a measure of the resistance to initial infection.

The data of the $96 \mathrm{DH}$ lines for resistance to spread of FHB in the host originated from Buerstmayr and associates (2002). Resistance to spread was determined using single-spikelet inoculation as described before (Buerstmayr et al. 2002). The experiments were conducted in 1999 and 2000 at the experimental field in Tulln with $F$. graminearum and $F$. culmorum strains (IFA65 and IFA104, respectively). Absolute number of bleached spikelets from the inoculation point downward was recorded for 10 treated heads. In addition, the percentage of inoculated ears with wilting symptoms was assessed. In wilted heads, the part of the spike above the point of inoculation completely wilts due to blockage of water and nutrient supplies.

Total FHB resistance was determined during two growth seasons (2002 and 2003) at the second location in Szeged, Hungary. The experiment again had a split-plot design with two replicates $\left(2 \mathrm{~m}^{2}\right)$, and the same isolates were used as in Tulln for the determination of total FHB resistance (IFA205 and IFA211). In Szeged, spring wheat routinely is planted in the middle of October. Preparation of inoculum was done according to Mesterházy (1995). Inoculations were made at middle anthesis by spraying 15 to 20 heads within the plot with the Fusarium suspension. High humidity was secured by polyethylene bag cover for $48 \mathrm{~h}$. Fusarium resistance (percentage of the diseased spikelets) was recorded at 10, 14, 18, 22, and 26 days after inoculation on a linear scale of 0 to $100 \%$.

\section{DON resistance test.}

DON (purity $>98 \%$ ) was purified at the IFA-Tulln according to a modified procedure published by Altpeter and Posselt (1994). DON resistance in the DH lines was tested in the greenhouse in the winter seasons of 2003 and 2004. For every DH line, two pots were sown with five plants each. Experimental design was a completely randomized block with two replications. During the flowering period, plant growth conditions were as follows: day and night temperatures of 20 and $18^{\circ} \mathrm{C}$, respectively; relative air humidity, $60 \%$; and illumination for $16 \mathrm{~h}$ at $15,000 \mathrm{~lx}$ at canopy height with two types of lamps, MF400BUH $\left(38,000 \mathrm{~lm} / \mathrm{m}^{2}\right)$ and NH360FLX $(47,800$ $\mathrm{lm} / \mathrm{m}^{2}$ ) (both from Iwasaki Electric Co. Ltd., Tokyo).

Wheat ears in the early flowering stage with protruding anthers in the center of the ear were selected. Two adjacent spikelets in the center of the ear were marked by clipping off a small part of the tips of the glumes with scissors. Each of the outer florets of both spikelets were treated with $20 \mu \mathrm{l}$ of the treatment solution, applied between the palea and lemma. In all, four outer florets were treated with $20 \mu \mathrm{l}$ of the test solution, resulting in the application of $80 \mu \mathrm{l}$ per ear. Test plants were treated with DON at $10 \mathrm{~g} \mathrm{liter}^{-1}, 0.1 \%$ Tween 20 followed $24 \mathrm{~h}$ later with DON at $2 \mathrm{~g} \mathrm{liter}^{-1}, 0.1 \%$ Tween 20. Control plants were treated with $0.1 \%$ Tween 20 at both time points. In all, 10 ears per wheat line (5 ears/pot, two replications) were treated with toxin and 6 ears (3 ears/pot, two replications) with 
the control solution. During and $2 \mathrm{~h}$ after treatment, the relative air humidity in the growth cabinet was increased to $80 \%$.

The number of DON-bleached spikelets (NDBS) in the basipetal direction, including the treated spikelets, was assessed at days $7,10,13$, and 16 after the first treatment. For each pot, mean NDBS of the five toxin-treated ears was used for the calculation of the AUDPC and for further statistical analyses. Treated ears were harvested after ripening. For toxin analyses, the DON-treated ears and the control ears of randomly selected DH lines were collected and ground separately.

\section{DON and DON-3-O-glucoside analyses.}

DON-3-O-glucoside and DON-15-glucoside were synthesized in a two-step reaction from 1 - $\beta$-bromo-1-deoxy-2,3,4,6tetra- $O$-acetyl- $\alpha$-D-gluco-pyranose and 15 -acetyl-DON or 3acetyl-DON as described (Berthiller et al. 2003; Savard 1991). The identity of the DON-3-O-glucoside was checked by full scan mass spectrometry (MS), MS/MS, and nuclear magnetic resonance spectroscopy (data not shown). DON-3-O-glucoside was purified further in a small scale with high-performance liquid chromatography (HPLC) at $25^{\circ} \mathrm{C}$ using methanol/water (10/90, vol/vol) on an RP-18 Aquasil column (Thermo Electron Corporation, Bellefonte, PA, U.S.A.) (100 by $4.6 \mathrm{~mm}$ i.d., $3 \mu \mathrm{m})$ and used as analytical standard. All organic solvents were purchased from J. T. Baker (Deventer, The Netherlands). Water was purified successively by reverse osmosis and a Milli-Q plus system from Millipore (Molsheim, France). The concentration of DON-3-O-glucoside in the standard solution was estimated from UV absorbance at $220 \mathrm{~nm}$, using the same molar extinction coefficients for DON and DON-3-O-glucoside. For each investigated DH line, $5.00 \pm 0.01 \mathrm{~g}$ of ground whole wheat ears was extracted for $90 \mathrm{~min}$ at $180 \mathrm{rpm}$ on an orbital shaker with $40 \mathrm{ml}$ of acetonitrile/water (84:16, vol/vol). The extracts were filtered, pressed through MycoSep 230 columns (Romer Labs Inc., Union, MO, U.S.A.) and $4 \mathrm{ml}$ of the cleaned extract were evaporated to dryness. The residue was dissolved in $1 \mathrm{ml}$ of methanol/water $(10: 90$, vol/vol), pressed through $0.45-\mu \mathrm{m}$ membrane filters, and analyzed by liquid chromatography (LC)-MS/MS.

LC-MS/MS analyses were performed with a QTrap-LCMS/MS system (Applied Biosystems, Foster City, CA, U.S.A.), equipped with an atmospheric pressure chemical ionization (APCI) source and an 1100 series HPLC system (Agilent, Waldbronn, Germany), including an 1100 series diode array detector. Chromatographic separation was achieved at $22^{\circ} \mathrm{C}$ using methanol/water $(15 / 85, \mathrm{vol} / \mathrm{vol})$ on an RP-18 Aquasil column (Thermo-Keystone) (100 by $4.6 \mathrm{~mm}$ i.d., 3 $\mu \mathrm{m})$. Injection volume was $50 \mu \mathrm{l}$ and flow rate was $0.8 \mathrm{ml} / \mathrm{min}$. The APCI source was operated at $450^{\circ} \mathrm{C}$ in negative ionization mode. Further MS parameters were curtain gas, 35 psi (241 $\mathrm{kPa}$ ); nebulizer gas, $60 \mathrm{psi}(414 \mathrm{kPa})$; auxiliary gas, 15 psi (103 kPa); corona discharge needle current, $-2 \mu \mathrm{A}$; collisionactivated dissociation gas, 6 (arbitrary units); multiple reaction monitoring (MRM) dwell time, $25 \mathrm{~ms}$; and pause between mass ranges, $5 \mathrm{~ms}$. Product ion spectra were gained at a collision energy of $-30 \mathrm{eV}$ and a declustering potential of $-46 \mathrm{~V}$. MRM transitions of $\mathrm{m} / \mathrm{z} 457.3$ to 295.3 for DON-3-O-glucoside and $\mathrm{m} / \mathrm{z} 295.3$ to 265.3 for DON were used. For quantification, the MRM mode and the UV signal at $220 \mathrm{~nm}$ were used, whereas enhanced product ion and MS/MS/MS (data not shown) scans were used to confirm the identity of DON-3-Oglucoside.

\section{Statistics.}

Individual assessment data as well as AUDPC data were used for further analyses. Variance analyses and $t$ tests (ANOVA and general linear model) as well as correlation analy- ses were carried out with SAS/STAT, version 6 (SAS Institute, Inc. 1989). In all cases, Spearman rank correlation coefficients were calculated.

QTL analyses were done by scanning across all linkage groups with the DON resistance data for the 96 wheat lines as described before (Buerstmayr et al. 2003). Genotyping of the DH lines was performed using 28 restriction fragment length polymorphisms, 267 amplified fragment length polymorphisms, 112 SSR, three storage proteins, and one morphological marker. In all, 387 polymorphic markers were mapped and they formed 40 linkage groups, covering a total genetic distance of 2,404 cM. The chromosome identity for 28 linkage groups was determined based on anchor microsatellite markers. SIM and CIM were carried out using the PLABQTL software (Utz and Melchinger 1996). Interval analyses of QTL for total FHB resistance and resistance to spread of FHB on the linkage group corresponding to chromosome $3 \mathrm{~B}$ were done with original resistance data as described previously (Buerstmayr et al. 2002, 2003). A QTL was considered significant with a logarithm of odds $>3$. For CIM, cofactor selection was done automatically with an $F$-to-enter threshold of 12 .

\section{ACKNOWLEDGMENTS}

This project is supported by the European Commission under the 5th Framework program. The contents of these pages are the sole responsibility of the publishers. These pages in no way represent the view of the Commission or its Services. We thank F. Doohan, J. A. Mullen, and D. Steele for the help with the preparation of the manuscript and the fruitful discussions. We thank Biopure Referenzsubstanzen $\mathrm{GmbH}$, Austria, for the technical assistance in the purification of DON. This work was financed by i) the European Union Research and Development Project "Novel tools for developing Fusarium resistant and toxin free wheat for Europe," contract number QLRT-2000-02044; ii) the pilot project "Fusarium virulence and plant resistance mechanisms" of the Austrian genome program GEN-AU; and iii) the Christian Doppler Research Association (CD-Laboratory of Mycotoxin Research at IFA Tulln).

\section{LITERATURE CITED}

Altpeter, F., and Posselt, U. K. 1994. Production of high quantities of 3 acetyldeoxynivalenol and deoxynivalenol. Appl. Microbiol. Biotechnol. 41:384-387.

Anderson, J. A., Stack, R. W., Liu, S., Waldron, B. L., Fjeld, A. D., Coyne, C., Moreno-Sevilla, B., Mitchell Fetch, J., Song, Q. J., Cregan, P. B., and Frohberg, R. C. 2001. DNA markers for Fusarium head blight resistance QTL in two wheat populations. Theor. Appl. Genet. 102:11641168

Bai, G.-H., Desjardins, A. E., and Plattner, R. D. 2001. Deoxynivalenolnonproducing Fusarium graminearum causes initial infection, but does not cause disease spread in wheat spikes. Mycopathologia 153:91-98.

Berthiller, F., Dall'Asta, C., Schuhmacher, R., Lemmens, M., Adam, G., and Krska, R. 2005. Masked mycotoxins: Determination of a deoxynivalenol glucoside in artificially and naturally contaminated wheat by LC-MS/MS J. Agric. Food Chem. 53:3421-3425.

Berthiller, F., Schuhmacher, R., Buttinger, G., Freudenschuss, G., Adam, G., and Krska, R. 2003. Synthesis of deoxynivalenol-glucosides and their characterization using a QTrap LC-MS/MS. Mycotoxin Res. 19:47-50.

Bottalico, A., and Perrone, G. 2002. Toxigenic Fusarium species and mycotoxins associated with head blight in small-grain cereals in Europe. Eur. J. Plant Pathol. 108:611-624.

Bowles, D. 2002. A multigene family of glycosyltransferases in a model plant, Arabidopsis thaliana. Biochem. Soc. Trans. 30:301-306.

Bruins, M. B. M., Karsai, I., Schepers, J., and Snijders, C. H. A. 1994. Phytotoxicity of deoxynivalenol to wheat tissue with regard to in vitro selection for Fusarium head blight resistance. Plant Sci. 94:195-206.

Buerstmayr, H., Hartl, L., Griesser, M., Angerer, N., Lengauer, D., Miedaner, T., Steiner, B., and Lemmens, M. 2003. Molecular mapping of QTL for Fusarium head blight resistance in wheat II. Combined type I and type II resistance. Theor. Appl. Genet. 107:503-508.

Buerstmayr, H., Lemmens, M., Hartl, L., Doldi, L., Steiner, B., Stierschneider, M., and Ruckenbauer, P. 2002. Molecular mapping of QTL for Fusarium head blight resistance in spring wheat I: Resistance 
to fungal spread (type II resistance). Theor. Appl. Genet. 104:84-91.

Gareis, W., Bauer, J., Thiem, J., Plank, G., Grabley, S., and Gedek, B. 1990. Cleavage of zearalenone-glycoside, a "masked" mycotoxin, during digestion in swine. J. Vet. Med. B 37:236-240.

Lemmens, M., Reisinger, A., Buerstmayr, H., and Ruckenbauer, P. 1994. Breeding for head blight (Fusarium spp.) resistance in wheat: Development of a mycotoxin-based selection method of seedlings. Acta Hortic. 355:223-232.

McCormick, S. 2003. The role of DON in pathogenicity. Pages 165-183 in: Fusarium Head Blight of Wheat and Barley. K. J. Leonard and W. R. Bushnell, eds. American Phytopathological Society Press, St. Paul, MN, U.S.A..

McLaughlin, C. S., Vaughn, M. H., Campbell, J. M., Wei, C. M., Stafford, M. E., and Hansin, B. S. 1977. Inhibition of protein synthesis by trichothecenes. Pages 263-273 in: Mycotoxins in Human and Animal Health, H. V. Rodricks, C. W. Hesseltine, and M. A. Mehlman, eds. Pathotoxin Publishers, Park Forest South, IL, U.S.A..

McMullen, M., Jones, R., and Gallenberg D. 1997. Scab of wheat and barley: A re-emerging disease of devastating impact. Plant Dis. 81:13401348.

Mesterházy, Á. 1995. Types and components of resistance to Fusarium head blight of wheat. Plant Breed. 114:377-386.

Mesterházy, Á. 2002. Role of deoxynivalenol in aggressiveness of Fusarium graminearum and $F$. culmorum in resistance to Fusarium head blight. Eur. J. Plant Pathol. 108:675-684.

Mesterházy, Á. 2003. Breeding wheat for Fusarium head blight resistance in Europe. Pages 211-240 in: Fusarium Head Blight of Wheat and Barley. K. J. Leonard and W. R. Bushnell, eds. American Phytopathological Society Press, St. Paul, MN, U.S.A..

Mesterházy, Á., Bartók, T., Mirocha, C. G., and Komoróczky, R. 1999. Nature of resistance to Fusarium head blight and the role of deoxynivalenol for breeding. Plant Breed. 118:97-110.

Miller, J. D., and Arnison, P. G. 1986. Degradation of deoxynivalenol by suspension cultures of the Fusarium head blight resistant wheat cultivar Frontana. Can. J. Plant Pathol. 8:147-150.

Miller, J. D., and Greenhalgh, R. 1988. Metabolites of fungal pathogens and plant resistance. Pages 117-129 in: Biotechnology for Crop Protection, Am. Chem. Soc. Symp. Ser. No. 379. P. A. Hedin, J. J. Menn, and R.M. Hollingworth, eds. American Chemical Society, U.S.A..

Parry, D. W., Jenkinson, P., and McLeod, L. 1995. Fusarium ear blight (scab) in small grain cereals-a review. Plant Pathol. 44:207-238.
Poppenberger, B., Berthiller, F., Lucyshyn, D., Sieberer, T., Schuhmacher, R., Krska, R., Kuchler, K., Glössl, J., Luschnig, C., and Adam, G. 2003. Detoxification of the Fusarium mycotoxin deoxynivalenol by a UDPglucosyltransferase from Arabidopsis thaliana. J. Biol. Chem. 278:47905-47914.

SAS Institute, Inc. 1989. SAS/STAT User's Guide, version 6, 4th ed. SAS Institute, Inc., Cary, NC, U.S.A.

Savard, M. E. 1991. Deoxynivalenol fatty acid and glucoside conjugates. J. Agric. Food Chem. 39:570-574.

Schroeder, H. W., and Christensen, J. J. 1963. Factors affecting resistance of wheat to scab caused by Gibberella zeae. Phytopathology 53:831838.

Shimada, T., and Otani, M. 1990. Effects of Fusarium mycotoxins on the growth of shoots and roots at germination of some Japanese wheat cultivars. Cereal Res. Commun. 18:229-232.

Sinha, R. C., and Savard, M. E. 1997. Concentration of deoxynivalenol in single kernels and various tissues of wheat heads. Can. J. Plant Pathol. 19:8-12.

Snijders, C. H. A. 1994. Breeding for resistance to Fusarium in wheat and maize. Pages 37-58 in: Mycotoxins in Grain. Compounds Other Than Aflatoxin. J. D. Miller and H. L. Trenholm, eds. Eagan Press, St. Paul, MN, U.S.A..

Somers, D. J., Fedak, G., and Savard, M. 2003. Molecular mapping of novel genes controlling Fusarium head blight resistance and deoxynivalenol accumulation in spring wheat. Genome 46:555-564.

Sutton, J. C. 1982. Epidemiology of wheat head blight and maize ear rot caused by Fusarium graminearum. Can. J. Plant Pathol. 4:195-209.

Utz, H. F., and Melchinger, A. E. 1996. PLABQTL: A program for composite interval mapping of QTL. J. Agr. Genomics. Published online.

Wakulinski, W. 1989. Phytotoxicity of the secondary metabolites of fungi causing wheat head fusariosis (head blight). Acta Physiol. Plant. 11:301-306.

Waldron, B. L., Moreno-Sevilla, B., Anderson, J. A., Stack, R. W., and Frohberg R. C. 1999. RFLP mapping of QTL for Fusarium head blight resistance in wheat. Crop Sci. 39:805-811.

Wang, Y. Z., and Miller, J. D. 1988. Effects of Fusarium graminearum metabolites on wheat tissue in relation to Fusarium head blight resistance. J. Phytopathol. 122:118-125.

Wojciechowski, S., Chelkowski, J., and Kostecki, M. 1995. Influence of deoxynivalenol on electrolyte leakage in cereal seedling leaves. Acta Physiol. Plant. 17:357-360. 